\title{
Synthesis of Functionalized Bicyclic Pyridones Containing the Dithiocarbamate Group Using Thioazlactones, Diamines, and Nitroketene Dithioacetal
}

\author{
Marziyeh Saeedia \\ Maryam Khoshdoun ${ }^{a}$ \\ Salman Taheri*a \\ Azim Ziyaei Halimehjani *b,c (iD) \\ Aliasghar Mohammadia \\ Mohammad Reza Halvagar ${ }^{a}$ \\ Vahid Amanid \\ a Chemistry \& Chemical Engineering Research Center of Iran, \\ P.O. Box 14335-186, Tehran, Iran \\ taherisalman@gmail.com \\ b Faculty of Chemistry, Kharazmi University, P. O. Box 15719-14911, \\ 49 Mofateh Street, Tehran, Iran \\ ziyaei@khu.ac.ir \\ ' Institut für Organische Chemie, Albert-Ludwigs-Universität Freiburg, \\ Albertstraße 21, 79104 Freiburg im Breisgau, Germany \\ d Department of Chemistry, Farhangian University, Tehran, Iran \\ Published as part of the Virtual Collection in Honor of Prof. Issa Yavari
}
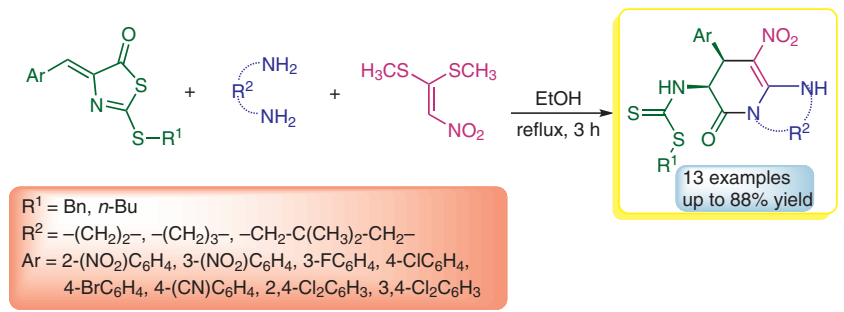

$4-\mathrm{BrC}_{6} \mathrm{H}_{4}, 4-(\mathrm{CN}) \mathrm{C}_{6} \mathrm{H}_{4}, 2,4-\mathrm{Cl}_{2} \mathrm{C}_{6} \mathrm{H}_{3}, 3,4-\mathrm{Cl}_{2} \mathrm{C}_{6} \mathrm{H}_{3}$

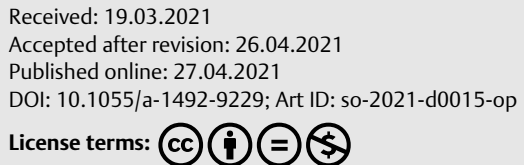

(c) 2021. The Author(s). This is an open access article published by Thieme under the terms of the Creative Commons Attribution-NonDerivative-NonCommercial-License, permitting copying and reproduction so long as the original work is given appropriate credit. Contents may not be used for commercial purposes or adapted, remixed, transformed or built upon. (https://creativecommons.org/licenses/by-nc-nd/4.0/)

Abstract An efficient method for the synthesis of highly substituted bicyclic pyridone derivatives containing the dithiocarbamate group is reported via a one-pot three-component reaction of 2-(alkylthio)thioazlactones, diamines, and nitroketene dithioacetal in EtOH under catalyst-free conditions. The reaction proceeds via a domino amidationintramolecular 1,4-addition-type Friedel-Crafts alkylation reaction to afford the corresponding fused bicyclic pyridones with high yields and diastereoselectivity.

Key words thioazlactone, nitroketene dithioacetal, bicyclic pyridine, dithiocarbamate, catalyst-free

Among the various types of fused heterocyclic compounds, bicyclic pyridone scaffolds are present in the structure of many biologically active compounds and natural products. ${ }^{1}$ Compounds containing these motifs show various properties including anticancer, ${ }^{2}$ antianxiety, ${ }^{3}$ antileishmanial, ${ }^{4}$ antibacterial, ${ }^{5}$ antifungal, ${ }^{6}$ anti-HIV, ${ }^{7}$ and antiinflammatory ${ }^{8}$ activities. Various methods for the synthesis of substituted bicyclic pyridones have been reported. ${ }^{9}$ Among them, the cyclocondensation of ketenaminals with dielectrophiles such as propiolic esters, ${ }^{10}$ diethyl but-2ynedioate, ${ }^{11} \beta$-ketoesterenol tosylates, ${ }^{12} \alpha$-bromoenals, ${ }^{13}$ azlactones, ${ }^{14}$ itaconic anhydride, ${ }^{15}$ and other compounds ${ }^{16}$ are extensively utilized for the synthesis of libraries of bicyclic pyridones.

Dithiocarbamates are well known for their applications as herbicides, fungicides, and pesticides in agriculture. ${ }^{17}$ The dithiocarbamate group is also a valuable pharmacophore that induces diverse biological activities when incorporated in a particular structure. ${ }^{18}$ Therefore, finding novel methods for the synthesis of various heterocycles containing a dithiocarbamate group is interesting for biological studies.

Heterocyclic ketene aminals are useful dinucleophiles for the construction of fused heterocyclic compounds and have been extensively applied in cascade reactions with dielectrophiles for the synthesis of condensed nitrogen-containing heterocycles. ${ }^{19}$

Although there are many reports on the utilization of azlactones in organic transformations,,$^{20}$ the use of thioazlactones with similar active sites has received less attention..$^{21}$ Lin and co-workers reported the synthesis of bicyclic pyridone derivatives using ketene aminals and azlactones in the presence of acetic acid. ${ }^{14}$ Recently, our group has reported a domino reaction for the diastereoselective synthesis of novel alkyl 2,3-dihydro-3-oxo-1-aryl-1H-benzo[f]chromen-2-ylcarbamodithioates and alkyl 3,4-dihydro-2-oxo-4-aryl-2H-chromen-3-ylcarbamodithioates via the reaction of naphthols and phenols with thioazlactones (Scheme 1, reaction 1). ${ }^{22}$ In continuation of our research toward the synthesis of novel dithiocarbamates and their applications as intermediates in organic chemistry, ${ }^{23}$ we wish 
to report herein the synthesis of novel bicyclic pyridone derivatives containing a dithiocarbamate group from 2-alkylthio-thioazlactones, diamines, and nitroketene dithioacetal (Scheme 1, reaction 2). ${ }^{24}$ The presence of the dithiocarbamate and pyridone motifs in a single skeleton may have a synergic effect to provide a new class of heterocyclic compounds with interesting biological activities.

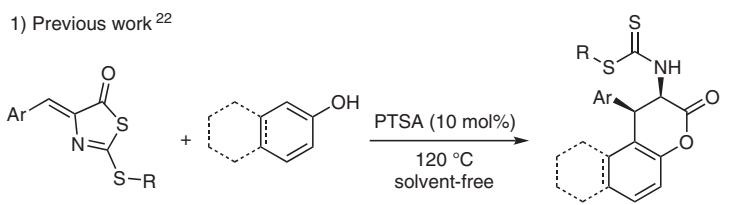

2) This work

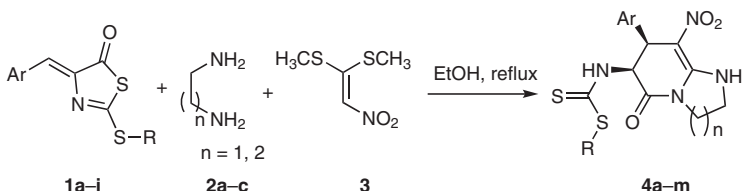

Scheme 1 Synthesis of novel dithiocarbamates and bicyclic pyridones containing a dithiocarbamate group

A model reaction between thioazlactone 1a (1 equiv), 1,3-diaminopropane $\mathbf{2 a}$ (1 equiv), and nitroketene dithioacetal 3 (1 equiv) was examined to optimize the reaction conditions. No reaction occurred at room temperature in water, $\mathrm{EtOH}$, and $\mathrm{CHCl}_{3}$ under catalyst-free conditions (Table 1 , entries $1-3$ ), nor was there any reaction in refluxing water (Table 1, entry 4). In refluxing EtOH for $3 \mathrm{~h}$ (Table 1, entry 5), a high yield (88\%) of the desired product $4 \mathbf{a}$ was obtained. Using an acid such as p-toluenesulfonic acid (Table 1 , entry 6 ) or a base such as triethylamine (Table 1 , entry 7) as possible catalysts for this reaction in ethanol showed no improvement of the reaction yield. In addition, varying other reaction conditions such as temperature, ratio of the starting materials, and reaction time did not improve the reaction yield.

After optimization of the reaction conditions, the scope and limitations of this protocol were examined using various thioazlactones and diamines in the reaction with nitroketene dithioacetal 3 (Table 2). We confirmed that thioazlactones with electron-withdrawing groups on the aryl group are suitable substrates for this protocol $(\mathbf{4 a}-\mathbf{m})$, but those containing an electron-donating group gave an inseparable mixture containing only trace amounts of the desired product. Varying the substitution pattern from benzylthio to butylthio on the thioazlactones gave similar yields (Table 2, entries 12 and 13 ). In addition, similar reactivities and yields were obtained using 1,2-diaminoethane, 1,3-diaminopropane, and 2,2-dimethyl-1,3-diaminopropane.
Table 1 Optimization of the Reaction Conditions for the Synthesis of Bicyclic Pyridones $4 \mathrm{a}^{\mathrm{a}}$

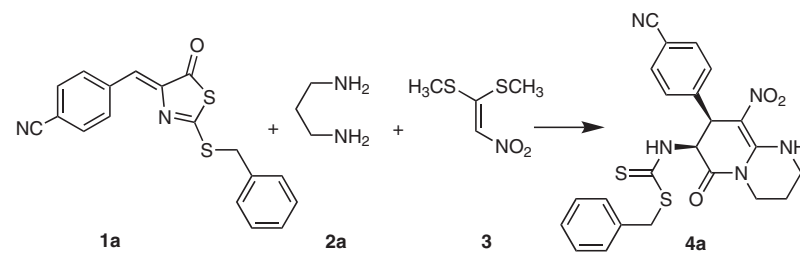

\begin{tabular}{lllll}
\hline Entry & Catalyst (mol\%) & Solvent & Temp $\left({ }^{\circ} \mathrm{C}\right)$ & ${\text { Yield }(\%)^{\mathrm{b}}}^{\circ}$ \\
\hline 1 & - & $\mathrm{EtOH}$ & r.t. & $\mathrm{NR}$ \\
2 & - & $\mathrm{CHCl}_{3}$ & r.t. & $\mathrm{NR}$ \\
3 & - & $\mathrm{H}_{2} \mathrm{O}$ & r.t. & $\mathrm{NR}$ \\
4 & - & $\mathrm{H}_{2} \mathrm{O}$ & reflux & $\mathrm{NR}$ \\
5 & - & $\mathrm{EtOH}$ & reflux & 88 \\
6 & PTSA (30) & $\mathrm{EtOH}$ & reflux & 64 \\
7 & Et $_{3} \mathrm{~N}(30)$ & EtOH & reflux & 45
\end{tabular}

a Reaction conditions: 1 a ( $1 \mathrm{mmol}), \mathbf{2 a}(1 \mathrm{mmol}), \mathbf{3}(1 \mathrm{mmol})$, and solvent (5 mL), $3 \mathrm{~h}$.

b Isolated yield.

Table 2 Regioselective Synthesis of Bicyclic Pyridones ${ }^{\mathrm{a}}$

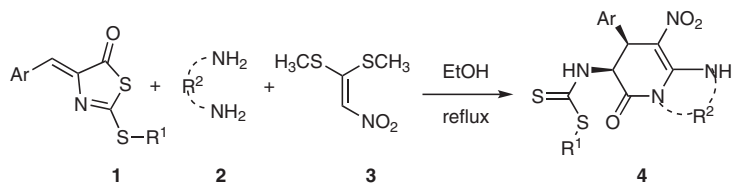

\begin{tabular}{|c|c|c|c|c|c|}
\hline Entry & $\mathrm{Ar}$ & $\mathrm{R}^{1}$ & $R^{2}$ & Product & Yield (\%) ${ }^{\mathrm{b}}$ \\
\hline 1 & 4- $\mathrm{NCC}_{6} \mathrm{H}_{4}$ & $\mathrm{Bn}$ & $-\left(\mathrm{CH}_{2}\right)_{3}-$ & $4 a$ & 88 \\
\hline 2 & $4-\mathrm{ClC}_{6} \mathrm{H}_{4}$ & $\mathrm{Bn}$ & $-\left(\mathrm{CH}_{2}\right)_{3}-$ & $4 b$ & 86 \\
\hline 3 & $2,4-\mathrm{Cl}_{2} \mathrm{C}_{6} \mathrm{H}_{4}$ & $\mathrm{Bn}$ & $-\left(\mathrm{CH}_{2}\right)_{3}-$ & $4 c$ & 83 \\
\hline 4 & $3,4-\mathrm{Cl}_{2} \mathrm{C}_{6} \mathrm{H}_{4}$ & $\mathrm{Bn}$ & $-\left(\mathrm{CH}_{2}\right)_{3}-$ & 4d & 85 \\
\hline 5 & $2-\mathrm{O}_{2} \mathrm{NC}_{6} \mathrm{H}_{4}$ & $\mathrm{Bn}$ & $-\left(\mathrm{CH}_{2}\right)_{3}-$ & $4 e$ & 80 \\
\hline 6 & $4-\mathrm{BrC}_{6} \mathrm{H}_{4}$ & $\mathrm{Bn}$ & $-\left(\mathrm{CH}_{2}\right)_{3}-$ & $4 f$ & 87 \\
\hline 7 & $3-\mathrm{FC}_{6} \mathrm{H}_{4}$ & $B n$ & $-\left(\mathrm{CH}_{2}\right)_{2}-$ & $4 g$ & 79 \\
\hline 8 & $3,4-\mathrm{Cl}_{2} \mathrm{C}_{6} \mathrm{H}_{4}$ & $\mathrm{Bn}$ & $-\left(\mathrm{CH}_{2}\right)_{2}-$ & $4 \mathrm{~h}$ & 84 \\
\hline 9 & $2,4-\mathrm{Cl}_{2} \mathrm{C}_{6} \mathrm{H}_{4}$ & $B n$ & $-\left(\mathrm{CH}_{2}\right)_{2}$ & $4 i$ & 82 \\
\hline 10 & $2-\mathrm{O}_{2} \mathrm{NC}_{6} \mathrm{H}_{4}$ & $\mathrm{Bn}$ & $-\mathrm{CH}_{2}-\mathrm{C}\left(\mathrm{CH}_{3}\right)_{2}-\mathrm{CH}_{2}-$ & $4 j$ & 78 \\
\hline 11 & $3-\mathrm{O}_{2} \mathrm{NC}_{6} \mathrm{H}_{4}$ & $\mathrm{Bn}$ & $-\mathrm{CH}_{2}-\mathrm{C}\left(\mathrm{CH}_{3}\right)_{2}-\mathrm{CH}_{2}-$ & $4 k$ & 80 \\
\hline 12 & $2,4-\mathrm{Cl}_{2} \mathrm{C}_{6} \mathrm{H}_{4}$ & $n-B u$ & $-\left(\mathrm{CH}_{2}\right)_{3}$ & $4 !$ & 87 \\
\hline 13 & $3,4-\mathrm{Cl}_{2} \mathrm{C}_{6} \mathrm{H}_{4}$ & $n-B u$ & $-\left(\mathrm{CH}_{2}\right)_{3}$ & $4 m$ & 89 \\
\hline
\end{tabular}

a Reaction conditions: 1 (1 mmol), 2 (1 mmol), 3 (1 mmol), in refluxing EtOH (5 mL) for $3 \mathrm{~h}$.

b Isolated yield. 
The structures of all desired products $\mathbf{4 a - m}$ were confirmed by FT-IR, ${ }^{1} \mathrm{H}$ NMR, and ${ }^{13} \mathrm{C}$ NMR spectroscopy and CHN analyses (see the Supporting Information). The ${ }^{1} \mathrm{H}$ NMR spectra showed four characteristic signals; two signals between $\delta=5.00-6.50 \mathrm{ppm}$ for the aliphatic $\mathrm{CH}$ resonances of the 3,4-dihydropyridone ring and two signals above $\delta=9.0 \mathrm{ppm}$ for the NH groups of the dithiocarbamate and enamine moieties. The ${ }^{13} \mathrm{C}$ NMR spectra also confirmed the product structure; the chemical shifts for the carbonyl groups of the pyridone ring being between $\delta=165-168$ ppm, and the dithiocarbamate group between $\delta=198-201$ $\mathrm{ppm}$. Unequivocal evidence for the structure of bicyclic pyridone 3f was obtained from single-crystal X-ray analysis (Figure 1). ${ }^{24}$ The same structure patterns were assumed for the other derivatives on the basis of their NMR spectroscopic similarities.

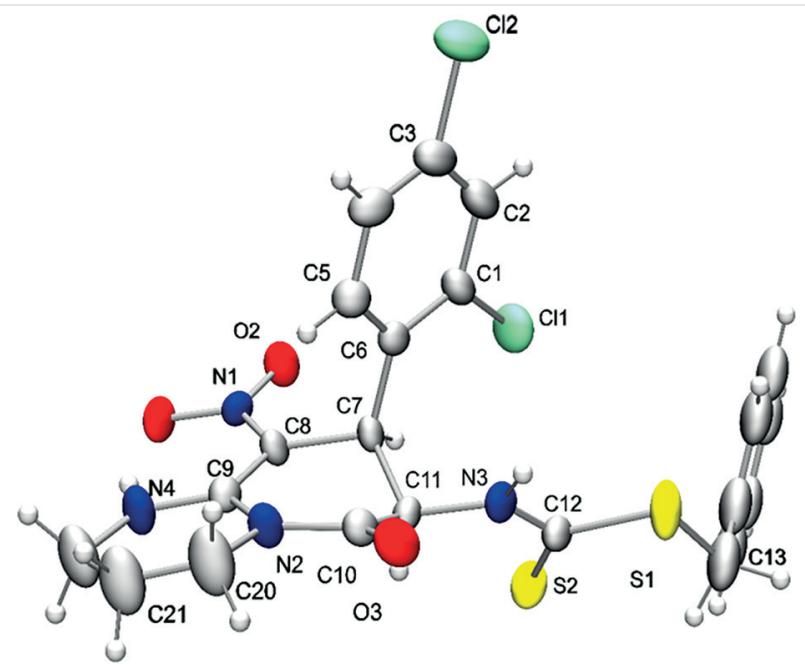

Figure 1 X-ray crystal structure of $\mathbf{4 c}$ (thermal ellipsoids set at the $40 \%$ probability level) $)^{24}$

A proposed mechanism for the formation of these bicyclic pyridones is presented in Scheme 2. The first event is condensation of diamine $\mathbf{2}$ and nitroketene dithioacetal $\mathbf{3}$ to give the isolable ketene aminal $\mathbf{5}$. According to the regioand stereochemistry of the products, it is conceivable that the next event is the ring opening of thioazlactone $\mathbf{1}$ with the nitrogen of ketene aminal $\mathbf{5}$ to afford intermediate $\mathbf{6}$ after tautomerism. Finally, an intramolecular Michael addition $^{25}$ provides the intermediate $\mathbf{7}$, followed by subsequent imine-enamine tautomerism to result in the desired product 4.

In summary, we have reported an efficient protocol for the synthesis of novel bicyclic pyridone derivatives via the reaction of 2-(alkylthio)thioazlactones, diamines, and nitroketene dithioacetal in $\mathrm{EtOH}$ under catalyst-free conditions. The reported method gives a straightforward route to

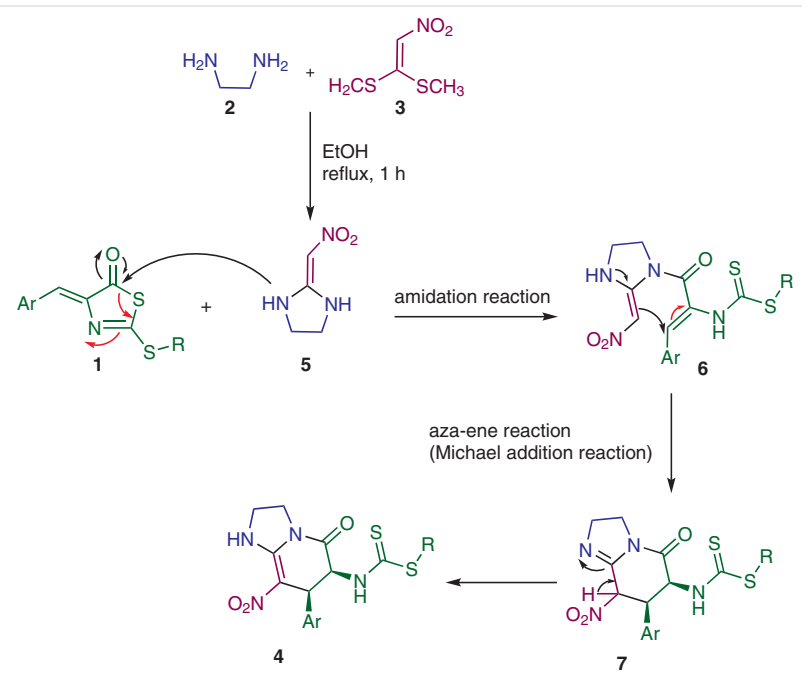

Scheme 2 A plausible mechanism for the regioselective formation of bicyclic pyridone derivatives 4

novel category of highly substituted bicyclic scaffolds containing pyridone and dithiocarbamate groups, both of which are interesting building blocks for biological studies.

Available reagents and solvents were purchased from commercial sources and were used without further purification. All ${ }^{1} \mathrm{H}$ NMR and ${ }^{13} \mathrm{C}$ NMR spectra were recorded on a Bruker $500 \mathrm{MHz} / 125 \mathrm{MHz}$ spectrometer in DMSO- $d_{6}$ and chemical shifts are reported in ppm. IR spectra were recorded on a Perkin-Elmer spectrum RX1 FT-IR spectrometer. Elemental analyses were conducted with a Perkin-Elmer 2400 Series II CHN analyzer. The X-ray diffraction measurement was carried out on a STOE IPDS-2T diffractometer with graphite-monochromated Mo Ka radiation. Column chromatography was performed with 230-400 mesh silica gel.

\section{General Procedure for the Preparation of Compounds 4a-m}

A mixture of diamine $(1 \mathrm{mmol})$ and nitroketene dithioacetal $(1 \mathrm{mmol})$ in EtOH was heated at reflux for $1 \mathrm{~h}$. Then, the thioazlactone ( $1 \mathrm{mmol}$ ) was added to the reaction mixture. Upon completion of the reaction after $2 \mathrm{~h}$, as indicated by TLC, the mixture was cooled to room temperature. The reaction mixture was filtered, and the residue was washed with hot water $(5 \mathrm{~mL})$ and then ethanol $(2 \mathrm{~mL})$ to give final pure product $\mathbf{4 a - m}$.

Benzyl \{8-(4-Cyanophenyl)-9-nitro-6-oxo-1,3,4,6,7,8-hexahydro2H-pyrido[1,2-a]pyrimidin-7-yl\}carbamodithioate (4a)

Colorless powder, mp $220-222^{\circ} \mathrm{C}$; yield $0.43 \mathrm{~g}(88 \%)$.

IR (KBr): $v_{\max }=3187,2976,2233,1720,1623,1325,1150 \mathrm{~cm}^{-1}$.

${ }^{1} \mathrm{H}$ NMR $\left(500 \mathrm{MHz}\right.$, DMSO- $\left.d_{6}\right): \delta=2.05-2.07(2 \mathrm{H}, m), 3.49(1 \mathrm{H}, m)$, $3.61(1 \mathrm{H}, m), 3.70(1 \mathrm{H}, m), 3.97(1 \mathrm{H}, m), 4.52\left(1 \mathrm{H}, d,{ }^{3} \mathrm{~J}=14 \mathrm{~Hz}, \mathrm{CH}_{2}\right)$, $4.56\left(1 \mathrm{H}, d,{ }^{3} \mathrm{~J}=14 \mathrm{~Hz}, \mathrm{CH}_{2}\right), 5.23\left(1 \mathrm{H}, d,{ }^{3} J=8.0 \mathrm{~Hz}, \mathrm{CH}\right), 5.93(1 \mathrm{H}, d d$, $J=8.0,7.5 \mathrm{~Hz}, \mathrm{CH}), 7.11\left(2 \mathrm{H}, d,{ }^{3} J=8.0 \mathrm{~Hz}, \mathrm{Ar}\right), 7.28\left(1 \mathrm{H}, t,{ }^{3} J=7.0 \mathrm{~Hz}\right.$, $\operatorname{Ar}), 7.35\left(2 \mathrm{H}, t,{ }^{3} J=7.5 \mathrm{~Hz}, \mathrm{Ar}\right), 7.39\left(2 \mathrm{H}, d,{ }^{3} J=7.45 \mathrm{~Hz}, \mathrm{Ar}\right), 7.70(2 \mathrm{H}$, $\left.d,{ }^{3} J=8.0 \mathrm{~Hz}, \mathrm{Ar}\right), 10.11\left(1 \mathrm{H}, d,{ }^{3} J=7.0 \mathrm{~Hz}, \mathrm{NH}\right), 11.45(1 \mathrm{H}, b r s, \mathrm{NH})$. 
${ }^{13} \mathrm{C}$ NMR $\left(125.7 \mathrm{MHz}\right.$, DMSO- $\left.d_{6}\right): \delta=19.7,39.4,40.4,40.8,40.9,60.0$, 107.9, 111.1, 119.6, 128.1, 129.3, 129.7, 130.0, 133.3, 137.9, 143.8, $152.5,167.1,198.9$

Anal. Calcd for $\mathrm{C}_{23} \mathrm{H}_{21} \mathrm{~N}_{5} \mathrm{O}_{3} \mathrm{~S}_{2}$ (479.57): C, 57.60; H, 4.41; N, 14.60 . Found: C, 57.80; H, 4.45; N, 14.75 .

\section{Benzyl\{8-(4-chlorophenyl)-9-nitro-6-oxo-1,3,4,6,7,8-hexahydro- 2H-pyrido[1,2-a]pyrimidin-7-yl\}carbamodithioate (4b) \\ Colorless powder, $\mathrm{mp} 220-222^{\circ} \mathrm{C}$; yield $0.420 \mathrm{~g}$ (86\%). \\ $\mathrm{IR}(\mathrm{KBr}): v_{\max }=3184,2980,1716,1619,1103 \mathrm{~cm}^{-1}$.}

${ }^{1} \mathrm{H}$ NMR $\left(500 \mathrm{MHz}\right.$, DMSO- $\left.d_{6}\right): \delta=2.04-2.06(2 \mathrm{H}, m), 3.48(1 \mathrm{H}, m)$, $3.63(1 \mathrm{H}, m), 3.69(1 \mathrm{H}, m), 3.96(1 \mathrm{H}, m), 4.51\left(1 \mathrm{H}, d,{ }^{3} \mathrm{~J}=13.0 \mathrm{~Hz}\right)$, $4.56\left(1 \mathrm{H}, d,{ }^{3} \mathrm{~J}=13.5 \mathrm{~Hz}\right), 5.16\left(1 \mathrm{H}, d,{ }^{3} \mathrm{~J}=7.5 \mathrm{~Hz}\right), 5.87(1 \mathrm{H}, d d, J=7.5$, $7.0 \mathrm{~Hz}), 6.93\left(2 \mathrm{H}, d,{ }^{3} \mathrm{~J}=8.2 \mathrm{~Hz}, \mathrm{Ar}\right), 7.28\left(3 \mathrm{H}, d,{ }^{3} \mathrm{~J}=8.1 \mathrm{~Hz}, \mathrm{Ar}\right), 7.34$ $\left(2 \mathrm{H}, t,{ }^{3} \mathrm{~J}=7.6 \mathrm{~Hz}, \mathrm{Ar}\right), 7.39\left(2 \mathrm{H}, d,{ }^{3} \mathrm{~J}=7.6 \mathrm{~Hz}, \mathrm{Ar}\right), 10.12\left(1 \mathrm{H}, d,{ }^{3} \mathrm{~J}=\right.$ $7.0 \mathrm{~Hz}, \mathrm{NH}), 11.45(1 \mathrm{H}$, br s, NH).

${ }^{13} \mathrm{C}$ NMR $\left(125.7 \mathrm{MHz}\right.$, DMSO- $\left.d_{6}\right): \delta=19.8,39.4,39.5,40.6,40.8,60.3$, 108.4, 128.0, 129.3, 129.3, 129.7, 130.7, 132.9, 136.8, 138.0, 152.5, $167.3,198.8$

Anal. Calcd for $\mathrm{C}_{22} \mathrm{H}_{21} \mathrm{ClN}_{4} \mathrm{O}_{3} \mathrm{~S}_{2}$ (489.01): C, 54.03; H, 4.33; N, 11.46. Found: C, 54.14; $\mathrm{H}, 4.37 ; \mathrm{N}, 11.22$.

Benzyl\{8-(2,4-dichlorophenyl)-9-nitro-6-oxo-1,3,4,6,7,8-hexahydro-2H-pyrido[1,2-a]pyrimidin-7-yl\}carbamodithioate (4c)

Colorless powder, mp $225-227^{\circ} \mathrm{C}$; yield $0.434 \mathrm{~g}$ (83\%).

IR $(\mathrm{KBr}): v_{\max }=3357,3205,1712,1623,1379 \mathrm{~cm}^{-1}$.

${ }^{1} \mathrm{H}$ NMR $\left(500 \mathrm{MHz}\right.$, DMSO- $\left.d_{6}\right): \delta=2.06-2.08(2 \mathrm{H}, m), 3.49(1 \mathrm{H}, m)$, $3.60(1 \mathrm{H}, m), 3.71(1 \mathrm{H}, m), 3.97(1 \mathrm{H}, m), 4.47\left(1 \mathrm{H}, d,{ }^{3} \mathrm{~J}=13.7 \mathrm{~Hz}\right)$, $4.61\left(1 \mathrm{H}, d,{ }^{3} \mathrm{~J}=13.7 \mathrm{~Hz}\right), 5.38\left(1 \mathrm{H}, d,{ }^{3} \mathrm{~J}=7.5 \mathrm{~Hz}\right), 6.23\left(1 \mathrm{H}, d d,{ }^{3} \mathrm{~J}=\right.$ 8.5, 7.5 Hz), 7.12 (1 H, $\left.d,{ }^{3} J=8.3 \mathrm{~Hz}, \mathrm{Ar}\right), 7.24-7.28(2 \mathrm{H}, \mathrm{m}, \mathrm{Ar}), 7.31(2$ $\left.\mathrm{H}, t,{ }^{3} J=7.6 \mathrm{~Hz}, \mathrm{Ar}\right), 7.37\left(2 \mathrm{H}, d,{ }^{3} \mathrm{~J}=7.27 \mathrm{~Hz}, \mathrm{Ar}\right), 7.51(1 \mathrm{H}, \mathrm{s}, \mathrm{Ar})$, $10.24\left(1 \mathrm{H}, d,{ }^{3} J=8.0 \mathrm{~Hz}, \mathrm{NH}\right), 11.42(1 \mathrm{H}$, br s, NH$)$.

${ }^{13} \mathrm{C}$ NMR $\left(125.7 \mathrm{MHz}\right.$, DMSO- $\left.d_{6}\right): \delta=19.3,31.1,37.1,39.0,40.3,59.4$, 108.4, 127.6, 128.0, 128.8, 128.8, 129.3, 130.4, 133.1, 134.7, 136.6, $137.7,152.3,166.8,200.0$.

Anal. Calcd for $\mathrm{C}_{22} \mathrm{H}_{20} \mathrm{Cl}_{2} \mathrm{~N}_{4} \mathrm{O}_{3} \mathrm{~S}_{2}$ (523.45): C, 50.48; H, 3.85; N, 10.70 . Found: C, 50.75; H, 3.92; N, 10.82 .

\section{Benzyl\{8-(3,4-dichlorophenyl)-9-nitro-6-oxo-1,3,4,6,7,8-hexa-} hydro-2H-pyrido[1,2-a]pyrimidin-7-yl\}carbamodithioate (4d) Colorless powder, mp $189-191{ }^{\circ} \mathrm{C}$; yield $0.445 \mathrm{~g}(85 \%)$.

IR $(\mathrm{KBr}): v_{\max }=3178,3003,1718,1617,1325,1149 \mathrm{~cm}^{-1}$.

${ }^{1} \mathrm{H}$ NMR $\left(500 \mathrm{MHz}\right.$, DMSO- $\left.d_{6}\right): \delta=2.04-2.06(2 \mathrm{H}, m), 3.47(1 \mathrm{H}, m)$, 3.60-3.70 (2 H, m), $3.99(1 \mathrm{H}, m), 4.51\left(1 \mathrm{H}, d,{ }^{3} J=13.8 \mathrm{~Hz}\right), 4.61(1 \mathrm{H}$, $\left.d,{ }^{3} J=13.8 \mathrm{~Hz}\right), 5.14\left(1 \mathrm{H}, d,{ }^{3} J=7.0 \mathrm{~Hz}\right), 5.90(1 \mathrm{H}, d d, J=7.5,7.5 \mathrm{~Hz})$, $6.91\left(1 \mathrm{H}, d d, J=1.9,8.3 \mathrm{~Hz}, 7.08(1 \mathrm{H}, s), 7.27\left(1 \mathrm{H}, t,{ }^{3} J=7.2 \mathrm{~Hz}, \mathrm{Ar}\right)\right.$, $7.34\left(2 \mathrm{H}, t,{ }^{3} J=7.6 \mathrm{~Hz}, \mathrm{Ar}\right), 7.39\left(2 \mathrm{H}, d,{ }^{3} J=7.3 \mathrm{~Hz}, \mathrm{Ar}\right), 7.48(1 \mathrm{H}, d$, $\left.{ }^{3} J=8.2 \mathrm{~Hz}, \mathrm{Ar}\right), 10.19\left(1 \mathrm{H}, d,{ }^{3} J=7.5 \mathrm{~Hz}, \mathrm{NH}\right), 11.45(1 \mathrm{H}, b r s, \mathrm{NH})$.

${ }^{13} \mathrm{C}$ NMR $\left(125.7 \mathrm{MHz}\right.$, DMSO- $\left.d_{6}\right): \delta=19.4,38.9,39.0,40.2,40.4,59.6$, 107.4, 127.6, 128.5, 128.9, 129.3, 130.6, 130.6, 131.1, 131.6, 137.6, 138.7, 152.1, 166.7, 198.6.

Anal. Calcd for $\mathrm{C}_{22} \mathrm{H}_{20} \mathrm{Cl}_{2} \mathrm{~N}_{4} \mathrm{O}_{3} \mathrm{~S}_{2}$ (523.45): C, 50.48; H, 3.85; N, 10.70 . Found: C, 50.67; H, 3.95; N, 10.91 .

Benzyl\{9-nitro-8-(2-nitrophenyl)-6-oxo-1,3,4,6,7,8-hexahydro2H-pyrido[1,2-a]pyrimidin-7-yl\}carbamodithioate (4e) Colorless powder, $\mathrm{mp} 210-213^{\circ} \mathrm{C}$; yield $0.399 \mathrm{~g}$ (80\%).
IR (KBr): $v_{\max }=3171,2999,1717,1615,1343 \mathrm{~cm}^{-1}$.

${ }^{1} \mathrm{H}$ NMR $\left(500 \mathrm{MHz}\right.$, DMSO- $\left.d_{6}\right): \delta=2.07(2 \mathrm{H}, m), 3.49(1 \mathrm{H}, m), 3.61(1$ $\mathrm{H}, m), 3.76(1 \mathrm{H}, m), 3.98(1 \mathrm{H}, m), 4.50\left(1 \mathrm{H}, d,{ }^{3} \mathrm{~J}=13.5 \mathrm{~Hz}\right), 4.56(1 \mathrm{H}$, $\left.d,{ }^{3} J=13.5 \mathrm{~Hz}\right), 5.61\left(1 \mathrm{H}, d,{ }^{3} \mathrm{~J}=8.0 \mathrm{~Hz}\right), 6.34(1 \mathrm{H}, d d, J=8.5,7.0 \mathrm{~Hz})$, 7.24-7.32 (4 H, m), $7.38\left(2 \mathrm{H}, d,{ }^{3} J=7.3 \mathrm{~Hz}, \mathrm{Ar}\right), 7.51\left(1 \mathrm{H}, t,{ }^{3} J=7.6 \mathrm{~Hz}\right.$, Ar), $7.60\left(1 \mathrm{H}, t,{ }^{3} J=7.4 \mathrm{~Hz}, \mathrm{Ar}\right), 7.85\left(1 \mathrm{H}, d,{ }^{3} \mathrm{~J}=7.9 \mathrm{~Hz}, \mathrm{Ar}\right), 10.27(1 \mathrm{H}$, $\left.d,{ }^{3} J=8.0 \mathrm{~Hz}, \mathrm{NH}\right), 11.42(1 \mathrm{H}, b r s, \mathrm{NH})$.

${ }^{13} \mathrm{C}$ NMR $\left(125.7 \mathrm{MHz}\right.$, DMSO- $\left.d_{6}\right): \delta=19.3,35.5,40.0,40.2,40.4,59.1$, $108.5,125.0,127.6,128.8,129.1,129.4,129.6,132.2,133.9,137.4$, 151.0, 152.2, 167.0, 200.0.

Anal. Calcd for $\mathrm{C}_{22} \mathrm{H}_{21} \mathrm{~N}_{5} \mathrm{O}_{5} \mathrm{~S}_{2}$ (499.56): C, 52.89; H, 4.24; N, 14.02 . Found: C, 53.01; H, 4.28; N, 14.15 .

Benzyl\{8-(4-bromophenyl)-9-nitro-6-oxo-1,3,4,6,7,8-hexahydro2H-pyrido[1,2-a]pyrimidin-7-yl\}carbamodithioate (4f)

Colorless powder, $\mathrm{mp} 195-197^{\circ} \mathrm{C}$; yield $0.464 \mathrm{~g}(87 \%)$.

IR (KBr): $v_{\max }=3184,2980,1716,1619,1103 \mathrm{~cm}^{-1}$.

${ }^{1} \mathrm{H}$ NMR (500 MHz, DMSO- $\left.d_{6}\right): \delta=2.04-2.06(2 \mathrm{H}, m), 3.48(1 \mathrm{H}, m)$, $3.63(1 \mathrm{H}, m), 3.68(1 \mathrm{H}, m), 3.96(1 \mathrm{H}, m), 4.52\left(1 \mathrm{H}, d,{ }^{3} \mathrm{~J}=13.7 \mathrm{~Hz}\right)$, $4.56\left(1 \mathrm{H}, d,{ }^{3} \mathrm{~J}=13.7 \mathrm{~Hz}\right), 5.15\left(1 \mathrm{H}, d,{ }^{3} \mathrm{~J}=7.5 \mathrm{~Hz}\right), 5.87(1 \mathrm{H}, d d, J=7.2$, $7.2 \mathrm{~Hz}), 6.87\left(2 \mathrm{H}, \mathrm{d},{ }^{3} \mathrm{~J}=8.1 \mathrm{~Hz}\right), 7.28\left(1 \mathrm{H}, d,{ }^{3} \mathrm{~J}=7.0 \mathrm{~Hz}, \mathrm{Ar}\right), 7.34(2 \mathrm{H}$, $\left.t,{ }^{3} J=7.5 \mathrm{~Hz}, \mathrm{Ar}\right), 7.41(4 \mathrm{H}, t, J=8.5 \mathrm{~Hz}, \mathrm{Ar}), 10.14\left(1 \mathrm{H}, d,{ }^{3} J=7.0 \mathrm{~Hz}\right.$, $\mathrm{NH}), 11.46(1 \mathrm{H}, b r s, \mathrm{NH})$.

${ }^{13} \mathrm{C}$ NMR $\left(125.7 \mathrm{MHz}\right.$, DMSO- $\left.d_{6}\right): \delta=19.4,31.1,40.2,40.4,40.6,59.9$, 108.0, 121.1, 127.6, 128.9, 129.4, 130.7, 131.8, 136.9, 137.6, 152.1, 166.9, 198.4 .

Anal. Calcd for $\mathrm{C}_{22} \mathrm{H}_{21} \mathrm{BrN}_{4} \mathrm{O}_{3} \mathrm{~S}_{2}$ (533.46): C, 49.53; $\mathrm{H}, 3.97 ; \mathrm{N}, 10.50$. Found: C, 49.84; H, 4.03; N, 10.62 .

Benzyl\{7-(3-fluorophenyl)-8-nitro-5-oxo-1,2,3,5,6,7-hexahydroimidazo[1,2-a]pyridin-6-yl\}carbamodithioate (4g)

Colorless powder, $\mathrm{mp} 209-211^{\circ} \mathrm{C}$; yield $0.362 \mathrm{~g}$ (79\%).

IR (KBr): $v_{\max }=3184,2980,1716,1619,1103 \mathrm{~cm}^{-1}$.

${ }^{1} \mathrm{H}$ NMR $\left(500 \mathrm{MHz}\right.$, DMSO- $\left.d_{6}\right): \delta=3.77(1 \mathrm{H}, m), 3.89(1 \mathrm{H}, m)$, 3.94$4.05(2 \mathrm{H}, \mathrm{m}), 4.50\left(1 \mathrm{H}, d,{ }^{3} \mathrm{~J}=14 \mathrm{~Hz}\right), 4.58\left(1 \mathrm{H}, d,{ }^{3} \mathrm{~J}=14 \mathrm{~Hz}\right), 5.12(1$ $\left.\mathrm{H}, d,{ }^{3} \mathrm{~J}=7.7 \mathrm{~Hz}\right), 5.86\left(1 \mathrm{H}, d,{ }^{3} \mathrm{~J}=7.0 \mathrm{~Hz}\right), 6.74\left(1 \mathrm{H}, d,{ }^{3} \mathrm{~J}=10 \mathrm{~Hz}\right), 6.79$ $\left(1 \mathrm{H}, d,{ }^{3} \mathrm{~J}=7.5 \mathrm{~Hz}, \mathrm{Ar}\right), 7.07\left(1 \mathrm{H}, t,{ }^{3} \mathrm{~J}=8.5 \mathrm{~Hz}, \mathrm{Ar}\right), 7.25-7.29(2 \mathrm{H}, m$, Ar), $7.33\left(2 \mathrm{H}, t,{ }^{3} J=7.6 \mathrm{~Hz}, \mathrm{Ar}\right), 7.38\left(2 \mathrm{H}, d,{ }^{3} \mathrm{~J}=7.3 \mathrm{~Hz}, \mathrm{Ar}\right), 9.81(1 \mathrm{H}$, br $s, \mathrm{NH}), 10.22(1 \mathrm{H}$, br $s, \mathrm{NH})$.

${ }^{13} \mathrm{C}$ NMR (125.7 MHz, DMSO- $d_{6}$ ): $\delta=19.0,37.6,43.9,44.7,60.9,106.0$, $115.1,127.6,127.9,128.0,128.8,129.2,129.7,131.0,132.4,138.0$, $152.8,166.1,199.1$

Anal. Calcd for $\mathrm{C}_{21} \mathrm{H}_{19} \mathrm{FN}_{4} \mathrm{O}_{3} \mathrm{~S}_{2}$ (458.53): C, 55.01; H, 4.18; N, 12.22. Found: C, 55.12; H, 4.21; N, 12.28 .

Benzyl\{7-(3,4-dichlorophenyl)-8-nitro-5-oxo-1,2,3,5,6,7-hexahydroimidazo[1,2-a]pyridin-6-yl\}carbamodithioate (4h):

Colorless powder, $\mathrm{mp} 210-212{ }^{\circ} \mathrm{C}$; yield $0.428 \mathrm{~g}$ (84\%).

IR (KBr): $v_{\max }=3324,1706,1637,1330 \mathrm{~cm}^{-1}$.

${ }^{1} \mathrm{H}$ NMR $\left(500 \mathrm{MHz}\right.$, DMSO- $\left.d_{6}\right): \delta=3.86-4.04(3 \mathrm{H}, m)$, $4.11(1 \mathrm{H}, \mathrm{m})$, $4.41\left(1 \mathrm{H}, d,{ }^{3} \mathrm{~J}=14.0 \mathrm{~Hz}\right), 4.48\left(1 \mathrm{H}, d,{ }^{3} \mathrm{~J}=14.0 \mathrm{~Hz}\right), 5.30\left(1 \mathrm{H}, d,{ }^{3} \mathrm{~J}=8\right.$ $\mathrm{Hz}), 5.71\left(1 \mathrm{H}, d,{ }^{3} \mathrm{~J}=7.5 \mathrm{~Hz}\right), 6.77\left(1 \mathrm{H}, d,{ }^{3} \mathrm{~J}=7.0 \mathrm{~Hz}\right), 6.98(1 \mathrm{H}, s)$, 7.16-7.24 (4 H, $m, \mathrm{Ar}), 7.28\left(2 \mathrm{H}, t,{ }^{3} \mathrm{~J}=7.5 \mathrm{~Hz}, \mathrm{Ar}\right), 8.37(1 \mathrm{H}, b r \mathrm{sH})$, $9.17(1 \mathrm{H}, b r s, \mathrm{NH})$.

${ }^{13} \mathrm{C}$ NMR (125.7 MHz, DMSO- $d_{6}$ ): $\delta=19.0,37.6,43.7,43.9,60.1,105.9$, 127.7, 127.9, 128.8, 129.3, 129.9, 130.9, 132.0, 132.8, 136.6, 137.5, $151.7,165.5,199.4$ 
Anal. Calcd for $\mathrm{C}_{21} \mathrm{H}_{18} \mathrm{Cl}_{2} \mathrm{~N}_{4} \mathrm{O}_{3} \mathrm{~S}_{2}$ (509.42): C, 49.51; H, 3.56; N, 11.00. Found: $\mathrm{C}, 49.58 ; \mathrm{H}, 3.60 ; \mathrm{N}, 11.08$.

Benzyl\{7-(2,4-dichlorophenyl)-8-nitro-5-oxo-1,2,3,5,6,7-hexahydroimidazo[1,2-a]pyridin-6-yl\}carbamodithioate (4i)

Colorless powder, mp $192-194{ }^{\circ} \mathrm{C}$; yield $0.428 \mathrm{~g}$ (84\%).

IR (KBr): $v_{\max }=3358,3205,1712,1623,1379 \mathrm{~cm}^{-1}$.

${ }^{1} \mathrm{H}$ NMR $\left(500 \mathrm{MHz}\right.$, DMSO- $\left.d_{6}\right): \delta=3.41-3.47(2 \mathrm{H}, \mathrm{m}), 3.96-4.03(2 \mathrm{H}$, $m), 4.48\left(1 \mathrm{H}, d,{ }^{3} \mathrm{~J}=13.5 \mathrm{~Hz}\right), 4.50\left(1 \mathrm{H}, \mathrm{d},{ }^{3} \mathrm{~J}=13.5 \mathrm{~Hz}\right), 5.33(1 \mathrm{H}, d$, $\left.{ }^{3} J=7.5 \mathrm{~Hz}\right), 6.23(1 \mathrm{H}, \mathrm{dd}, J=8.0,7.5 \mathrm{~Hz}), 7.19\left(1 \mathrm{H}, d,{ }^{3} J=8.4 \mathrm{~Hz}, \mathrm{Ar}\right)$, 7.24-7.28 (2 H, $m$, Ar), $7.31\left(2 \mathrm{H}, t,{ }^{3} J=7.5 \mathrm{~Hz}, \mathrm{Ar}\right), 7.36\left(2 \mathrm{H}, d,{ }^{3} \mathrm{~J}=7.5\right.$ $\mathrm{Hz}, \mathrm{Ar}), 7.51(1 \mathrm{H}, s, \mathrm{Ar}), 9.83(1 \mathrm{H}, b r s, \mathrm{NH}), 10.24\left(1 \mathrm{H}, d,{ }^{3} \mathrm{~J}=8.0 \mathrm{~Hz}\right.$, $\mathrm{NH})$.

${ }^{13} \mathrm{C}$ NMR (125.7 MHz, DMSO- $\left.d_{6}\right): \delta=19.0,37.6,44.4,56.5,60.0,106.0$, 127.6, 128.1, 128.8, 129.3, 129.4, 132.8, 133.1, 135.1, 136.4, 137.7, 152.6, 165.5, 200.0.

Anal. Calcd for $\mathrm{C}_{21} \mathrm{H}_{18} \mathrm{Cl}_{2} \mathrm{~N}_{4} \mathrm{O}_{3} \mathrm{~S}_{2}$ (509.42): C, 49.51; H, 3.56; N, 11.00. Found: C, 49.58; H, 3.60; N, 11.08 .

\section{Benzyl\{3,3-dimethyl-9-nitro-8-(2-nitrophenyl)-6-oxo-1,3,4,6,7,8-} hexahydro-2H-pyrido[1,2-a]pyrimidin-7-yl\}carbamodithioate (4j) Colorless powder, mp $199-202{ }^{\circ} \mathrm{C}$; yield $0.411 \mathrm{~g}$ (78\%).

IR (KBr): $v_{\max }=3187,1719,1623,1528 \mathrm{~cm}^{-1}$.

${ }^{1} \mathrm{H}$ NMR (500 MHz, DMSO- $\left.d_{6}\right): \delta=1.07(3 \mathrm{H}, m), 1.09(3 \mathrm{H}, m), 3.28(1$ $\mathrm{H}, m), 3.35(1 \mathrm{H}, m), 3.59\left(1 \mathrm{H}, d,{ }^{3} \mathrm{~J}=12.6 \mathrm{~Hz}\right), 3.70\left(1 \mathrm{H}, d,{ }^{3} \mathrm{~J}=12.6\right.$ $\mathrm{Hz}), 4.51\left(1 \mathrm{H}, d,{ }^{3} \mathrm{~J}=13.6 \mathrm{~Hz}\right), 4.57\left(1 \mathrm{H}, d,{ }^{3} J=13.6 \mathrm{~Hz}\right), 5.64(1 \mathrm{H}, d$, $\left.{ }^{3} J=7.8 \mathrm{~Hz}\right), 6.41(1 \mathrm{H}, d d, J=7.9,7.9 \mathrm{~Hz}), 7.22-7.27(2 \mathrm{H}, m, \mathrm{Ar}), 7.31$ $\left(2 \mathrm{H}, t,{ }^{3} \mathrm{~J}=7.4 \mathrm{~Hz}, \mathrm{Ar}\right), 7.38\left(2 \mathrm{H}, d,{ }^{3} \mathrm{~J}=7.3 \mathrm{~Hz}, \mathrm{Ar}\right), 7.51\left(1 \mathrm{H}, t,{ }^{3} \mathrm{~J}=7.5\right.$ $\mathrm{Hz}, \mathrm{Ar}), 7.61\left(1 \mathrm{H}, t,{ }^{3} J=7.5 \mathrm{~Hz}, \mathrm{Ar}\right), 7.87\left(1 \mathrm{H}, d,{ }^{3} J=8.0 \mathrm{~Hz}, \mathrm{Ar}\right), 10.27$ $\left(1 \mathrm{H}, d,{ }^{3} J=8.0 \mathrm{~Hz}, \mathrm{NH}\right), 11.38(1 \mathrm{H}, b r s, \mathrm{NH})$.

${ }^{13} \mathrm{C}$ NMR $\left(125.7 \mathrm{MHz}\right.$, DMSO- $\left.d_{6}\right): \delta=23.8,24.2,27.4,35.6,39.3,50.6$, 50.8, 59.0, 108.5, 125.1, 127.6, 128.8, 129.1, 129.3, 129.4, 132.2, 133.8, 137.4, 151.1, 151.1, 167.3, 200.2.

Anal. Calcd for $\mathrm{C}_{24} \mathrm{H}_{25} \mathrm{~N}_{5} \mathrm{O}_{5} \mathrm{~S}_{2}$ (527.61): C, 54.63; $\mathrm{H}, 4.78 ; \mathrm{N}, 13.27$. Found: $\mathrm{C}, 54.95 ; \mathrm{H}, 4.93 ; \mathrm{N}, 13.56$.

\section{Benzyl\{3,3-dimethyl-9-nitro-8-(3-nitrophenyl)-6-oxo-1,3,4,6,7,8- hexahydro-2H-pyrido[1,2-a]pyrimidin-7-yl\}carbamodithioate (4k)}

Colorless powder, mp $216-218{ }^{\circ} \mathrm{C}$; yield $0.422 \mathrm{~g}$ (80\%).

IR (KBr): $v_{\max }=3187,1719,1623,1528 \mathrm{~cm}^{-1}$.

${ }^{1} \mathrm{H}$ NMR $\left(500 \mathrm{MHz}\right.$, DMSO- $\left.d_{6}\right): \delta=1.10(3 \mathrm{H}, s), 1.12(3 \mathrm{H}, s), 3.24(1 \mathrm{H}$, $m), 3.42(1 \mathrm{H}, m), 3.60(2 \mathrm{H}, m), 4.52\left(1 \mathrm{H}, d,{ }^{3} J=13.5 \mathrm{~Hz}\right), 4.58(1 \mathrm{H}, d$, $\left.{ }^{3} J=14 \mathrm{~Hz}\right), 5.33(1 \mathrm{H}, d, J=7.5 \mathrm{~Hz})$, ), $6.06(1 \mathrm{H}, d d, J=7.5,7.5 \mathrm{~Hz}), 7.27$ $\left(1 \mathrm{H}, t,{ }^{3} J=7.5 \mathrm{~Hz}, \mathrm{Ar}\right), 7.34\left(2 \mathrm{H}, t,{ }^{3} \mathrm{~J}=7.5 \mathrm{~Hz}, \mathrm{Ar}\right), 7.36-7.39(3 \mathrm{H}, m$, $\mathrm{Ar}), 7.57\left(1 \mathrm{H}, t,{ }^{3} J=8.0 \mathrm{~Hz}, \mathrm{Ar}\right), 7.72(1 \mathrm{H}, s, \mathrm{Ar}), 8.15(1 \mathrm{H}, d d, J=8.0$, $1.5 \mathrm{~Hz}, \mathrm{Ar}), 10.15\left(1 \mathrm{H}, d,{ }^{3} J=7.5 \mathrm{~Hz}, \mathrm{NH}\right), 11.4(1 \mathrm{H}, \operatorname{br~} s, \mathrm{NH})$.

${ }^{13} \mathrm{C}$ NMR $\left(125.7 \mathrm{MHz}\right.$, DMSO- $\left.d_{6}\right): \delta=24.3,24.3,27.9,39.4,40.4,50.9$, 51.5, 59.8, 107.8, 123.1, 123.5, 128.0, 129.3, 129.7, 131.0, 135.6, 137.8, $140.1,148.8,151.4,167.5,199.1$.

Anal. Calcd for $\mathrm{C}_{24} \mathrm{H}_{25} \mathrm{~N}_{5} \mathrm{O}_{5} \mathrm{~S}_{2}$ (527.61): C, 54.63; H, 4.78; N, 13.27. Found: C, 54.97; H, 5.02; N, 13.33 .

Butyl\{8-(2,4-dichlorophenyl)-9-nitro-6-oxo-1,3,4,6,7,8-hexahydro-2H-pyrido[1,2-a]pyrimidin-7-yl\}carbamodithioate (41) Colorless powder, mp $230-232^{\circ} \mathrm{C}$; yield $0.382 \mathrm{~g}$ (78\%).

IR $(\mathrm{KBr}): v_{\max }=3175,1718,1617,1325,1149 \mathrm{~cm}^{-1}$
${ }^{1} \mathrm{H}$ NMR $\left(500 \mathrm{MHz}\right.$, DMSO- $\left.d_{6}\right): \delta=0.90\left(3 \mathrm{H}, t,{ }^{3} \mathrm{~J}=7.3 \mathrm{~Hz}\right), 1.34-1.41$ $(2 \mathrm{H}, m), 1.56-1.62(2 \mathrm{H}, m), 2.07(2 \mathrm{H}, t, 3 J=5.6 \mathrm{~Hz}), 3.15(1 \mathrm{H}, m)$, $3.25(1 \mathrm{H}, m), 3.49(1 \mathrm{H}, m), 3.61(1 \mathrm{H}, m), 3.72(1 \mathrm{H}, m), 3.98(1 \mathrm{H}, m)$, $5.38\left(1 \mathrm{H}, d,{ }^{3} J=7.9 \mathrm{~Hz}\right), 6.23(1 \mathrm{H}, d d, J=7.8,7.8 \mathrm{~Hz}), 7.12(1 \mathrm{H}, d, J=$ $8.4 \mathrm{~Hz}, \mathrm{Ar}), 7.27(1 \mathrm{H}, d d, J=8.4,1.9 \mathrm{~Hz}, \mathrm{Ar}), 7.53\left(1 \mathrm{H}, d,{ }^{3} J=1.9 \mathrm{~Hz}\right.$, Ar), $10.0\left(1 \mathrm{H}, d,{ }^{3} J=8.0 \mathrm{~Hz}, \mathrm{NH}\right), 11.42(1 \mathrm{H}, b r s, \mathrm{NH})$.

${ }^{13} \mathrm{C}$ NMR $\left(125.7 \mathrm{MHz}\right.$, DMSO- $\left.d_{6}\right): \delta=14.0,19.3,21.8,31.3,31.4,34.5$, $34.6,37.1,59.1,108.4,128.0,129.4,130.4,133.0,134.8,136.6,152.3$, 166.9, 200.6.

Anal. Calcd for $\mathrm{C}_{19} \mathrm{H}_{22} \mathrm{Cl}_{2} \mathrm{~N}_{4} \mathrm{O}_{3} \mathrm{~S}_{2}$ (489.43): C, 46.63; $\mathrm{H}, 4.53$; N, 11.45 . Found: $\mathrm{C}, 46.85 ; \mathrm{H}, 4.67$; N, 11.63 .

Butyl\{8-(3,4-dichlorophenyl)-9-nitro-6-oxo-1,3,4,6,7,8-hexahydro-2H-pyrido[1,2-a]pyrimidin-7-yl\}carbamodithioate (4m) Colorless powder, mp $193-195{ }^{\circ} \mathrm{C}$; yield $0.386 \mathrm{~g}$ (79\%).

IR $(\mathrm{KBr}): v_{\max }=3175,3003,1718,1617,1325,1149 \mathrm{~cm}^{-1}$.

${ }^{1} \mathrm{H}$ NMR $\left(500 \mathrm{MHz}\right.$, DMSO- $\left.d_{6}\right): \delta=0.91\left(3 \mathrm{H}, t,{ }^{3} \mathrm{~J}=7.3 \mathrm{~Hz}\right), 1.36-1.43$ $(2 \mathrm{H}, m), 1.59-1.65(2 \mathrm{H}, m), 2.05\left(2 \mathrm{H}, t,{ }^{3} \mathrm{~J}=5.5 \mathrm{~Hz}\right), 3.20(1 \mathrm{H}, m)$, $3.28(1 \mathrm{H}, m), 3.49(1 \mathrm{H}, m), 3.61-3.69(2 \mathrm{H}, m), 3.99(1 \mathrm{H}, m), 5.14(1$ $\left.\mathrm{H}, d,{ }^{3} J=7.7 \mathrm{~Hz}\right), 5.90(1 \mathrm{H}, d d, J=7.4,7.4 \mathrm{~Hz}), 6.94\left(1 \mathrm{H}, d,{ }^{3} J=8.2 \mathrm{~Hz}\right.$, Ar), $7.07(1 \mathrm{H}, s, \mathrm{Ar}), 7.51\left(1 \mathrm{H}, d,{ }^{3} \mathrm{~J}=8.2 \mathrm{~Hz}, \mathrm{Ar}\right), 10.0\left(1 \mathrm{H}, d,{ }^{3} \mathrm{~J}=7.2\right.$ $\mathrm{Hz}, \mathrm{NH}), 11.45$ (1 H, br s, NH).

${ }^{13} \mathrm{C}$ NMR $\left(125.7 \mathrm{MHz}\right.$, DMSO- $\left.d_{6}\right): \delta=14.0,19.3,21.8,21.8,25.7,31.4$, 34.4, 39.1, 59.4, 107.4, 128.5, 130.6, 130.7, 131.1, 131.6, 138.8, 152.1, $166.7,199.2$

Anal. Calcd for $\mathrm{C}_{19} \mathrm{H}_{22} \mathrm{Cl}_{2} \mathrm{~N}_{4} \mathrm{O}_{3} \mathrm{~S}_{2}$ (489.43): C, 46.63; H, 4.53; N, 11.45. Found: C, 46.71; $\mathrm{H}, 4.60 ; \mathrm{N}, 11.72$.

\section{Conflict of Interest}

The authors declare no conflict of interest.

\section{Funding Information}

We thank the Research Council of the Chemistry and Chemical Engineering Research Center of Iran (CCERCI) and the Faculty of Chemistry of Kharazmi University for supporting this work. A.Z.H. thanks the Alexander von Humboldt-Stiftung for supporting his research in the Albert-Ludwigs-Universität Freiburg.

\section{Supporting Information}

Supporting information for this article is available online at https://doi.org/10.1055/a-1492-9229.

\section{References}

(1) (a) Taguchi, H.; Yazawa, H.; Arnett, J. F.; Kishi, Y. Tetrahedron Lett. 1977, 7, 627. (b) Shen, W.; Coburn, C. A.; Bornmann, W. G.; Danishefsky, S. J. Org. Chem. 1993, 58, 611. (c) Paulvannan, K.; Stille, J. R. J. Org. Chem. 1994, 59, 1613. (d) Aaberg, V.; Almqvist, F. Org. Biomol. Chem. 2007, 5, 1827. (e) Pinkner, J. S.; Remaut, H.; Miller, E.; Aaberg, V.; Pemberton, N.; Hedenstroem, M.; Larsson, A.; Seed, P.; Waksman, G.; Hultgren, S. J.; Almqvist, F. Proc. Natl. Acad. Sci. U.S.A. 2006, 103, 17897. (f) Marcaurelle, L. A.; Johannes, C.; Yohannes, D.; Tillotson, B. P.; Mann, D. Bioorg. Med. Chem. Lett. 2009, 19, 2500. 
(2) Yin, L.; Hu, Q.-Z.; Hartmann, L. W. J. Med. Chem. 2013, 56, 460.

(3) Collins, I.; Moyes, C.; Davey, W. B.; Rowley, M.; Bromidge, F. A.; Quirk, K.; Atack, J. R.; McKernan, R. M.; Thompson, S. A.; Wafford, K.; Dawson, G. R.; Pike, A.; Sohal, B.; Tsou, N. N.; Ball, R. G.; Castro, J. L. J. Med. Chem. 2002, 45, 1887.

(4) (a) Fan, X.; Feng, D.; Qu, Y.; Zhang, X.; Wang, J.; Loiseau, P. M.; Andrei, G.; Snoeck, R.; De Clercq, E. Bioorg. Med. Chem. Lett. 2010, 20, 809. (b) Hussain, H.; Al-Harrasi, A.; Al-Rawahi, A.; Green, I. R.; Gissons, S. Chem. Rev. 2014, 114, 10369.

(5) Flamm, R. K.; Vojtko, C.; Chu, D. T. W.; Beyer, Q. L. J.; Hensey, D.; Ramer, N.; Clement, J. J.; Tanaka, S. K. Antimicrob. Agents Chemother. 1995, 4, 964.

(6) Haga, A.; Tamoto, H.; Ishino, M.; Kimura, E.; Sugita, T.; Kinoshita, K.; Takahashi, K.; Shiro, M.; Koyama, K. J. Nat. Prod. 2013, 76, 750.

(7) Jones, E. D.; Vandegraaff, N.; Le, G.; Choi, N.; Issa, W.; Macfarlane, K.; Thienthong, N.; Winfield, L. J.; Coates, J. A. V.; Lu, L.; Li, X.-M.; Feng, B.; Yu, C.-J.; Rhodes, D. I.; Deadman, J. J. Bioorg. Med. Chem. Lett. 2010, 20, 5913.

(8) Amr, A. G.; Abdulla, M. M. Bioorg. Med. Chem. 2006, 14, 4341.

(9) (a) Zhao, M.-X.; Wang, M.-X.; Huang, Z.-T. Tetrahedron 2002, 58, 1309. (b) Kubo, K.; Ito, N.; Souzu, I.; Isomura, Y.; Homma, H.; Murakami, M. US4284778A, 1981. (c) Hehemann, D. G.; Winnik, W. J. Heterocycl. Chem. 1994, 31, 393. (d) Liao, W.-L.; Li, S.-Q.; Wang, J.; Zhang, Z.-Y.; Yang, Z.-W.; Xu, D.; Xu, C.; Lan, H.-T.; Chen, Z.-Z.; Xu, Z.-G. ACS Comb. Sci. 2016, 18, 65. (e) Kavala, V.; Wang, C. C.; Wang, Y. H.; Kuo, C. W.; Janreddy, D.; Huang, W. C.; Kuo, T. S.; He, C. H.; Chen, M. L.; Yao, C. F. Adv. Synth. Catal. 2014, 356, 2609. (f) Lu, J.; Gong, X.; Yang, H.; Fu, H. Chem. Commun. 2010, 46, 4172. (g) Sunke, R.; Adepu, R.; Kapavarapu, R.; Chintala, S.; Meda, C. L. T.; Parsa, K. V. L.; Pal, M. Chem. Commun. 2013, 49, 3570.

(10) Huang, Z.-T.; Liu, Z.-R. Heterocycles 1986, 24, 2247.

(11) Huang, Z.-T.; Tzai, L. H. Chem. Ber. 1986, 2208.

(12) Yan, S.-J.; Niu, Y.-F.; Huang, R.; Lin, J. Synlett 2009, 2821.

(13) Yao, C.; Jiao, W.; Xiao, Z.; Xie, Y.; Li, T.; Wang, X.; Liu, R.; Yu, C. RSC Adv. 2013, 3, 10801.

(14) Chen, X.; Zhu, D.; Wang, X.; Yan, S.; Lin, J. Tetrahedron 2013, 69, 9224.

(15) Ren, L.; Lou, Y.; Chen, N.; Xia, S.; Shao, X.; Xu, X.; Li, Z. Synth. Commun. 2014, 44, 858.

(16) (a) Zhao, M.-X.; Wang, M.-X.; Huang, Z.-T. Tetrahedron 2002, 58, 1309. (b) Meyer, H. Liebigs Ann. Chem. 1981, 9, 1523. (c) Takahashi, M.; Nozaki, C.; Shibazaki, Y. Chem. Lett. 1987, 6, 1229. (d) Raymond, C. F. J.; Pravin, P. Tetrahedron 1998, 54, 6191. (e) Charkrabarti, S.; Panda, K.; Misra, N. C.; Ila, H.; Junjappa, H. Synlett 2005, 1437. (f) Yu, C. Y.; Yang, P. H.; Zhao, M. X.; Huang, Z. T. Synlett 2006, 1835. (g) Sukach, V. A.; Bolbut, A. V.; Petin, A. Y.; Vovk, M. V. Synthesis 2007, 835.

(17) (a) Kanchi, S.; Singh, P.; Bisetty, K. Arab. J. Chem. 2014, 7, 11. (b) Hussein, M. A.; El-Shorbagi, A.-N.; Khallil, A.-R. Arch. Pharm. Med. Chem. 2001, 334, 305. (c) Eng, G.; Song, X.; Duong, Q.; Strickman, D.; Glass, J.; May, L. Appl. Organomet. Chem. 2003, 17, 218.

(18) (a) Kiran Kumar, S. T. V. S.; Kumar, L.; Sharma, V. L.; Jain, A.; Jain, R. K.; Maikhuri, J. P.; Kumar, M.; Shukla, P. K.; Gupta, G. J. Med. Chem. 2008, 43, 2247. (b) Kumar, L.; Lal, N.; Kumar, V.; Sarswat,
A.; Jangir, S.; Bala, V.; Kumar, L.; Kushwaha, B.; Pandey, A. K.; Siddiqi, M. I.; Shukla, P. K.; Maikhuri, J. P.; Gupta, G.; Sharma, V. L. Eur. J. Med. Chem. 2013, 70, 68. (c) Tripathi, R. P.; Khan, A. R.; Setty, B. S.; Bhaduri, A. P. Acta Pharm. 1996, 46, 169. (d) Ates, O.; Kocabalkanli, A.; Cesur, N.; Otuk, G. Farmaco 1998, 53, 541. (e) Nofal, Z. M.; Fahmy, H. H.; Mohamed, H. S. Arch. Pharm. Res. 2002, 25, 28. (f) Singh, N.; Gupta, S.; Nath, G. Appl. Organomet. Chem. 2000, 14, 484.

(19) (a) Cheng, Y.; Yang, H.-B.; Huang, Z.-T.; Wang, M.-X. Tetrahedron Lett. 2001, 42, 1757. (b) Nazarenko, K. G.; Shvidenko, K. V.; Pinchuk, A. M.; Tolmachev, A. A. Monatsh. Chem. 2005, 136, 211. (c) Zhou, A.; Pittman, C. U. Tetrahedron Lett. 2006, 46, 2045. (d) Huang, Z.-T.; Wang, M.-X. Heterocycles 1994, 37, 1233. (e) Yan, S.-J.; Chen, Y.-L.; Liu, L.; He, N.-Q.; Lin, J. Green Chem. 2010, 12, 2043. (f) Yu, F.-C.; Huang, R.; Ni, H.-C.; Fan, J.; Yan, S.J.; Lin, J. Green Chem. 2013, 15, 453. (g) Wen, L.-R.; Li, Z.-R.; Li, M.; Cao, H. Green Chem. 2012, 14, 707. (h) Li, M.; Shao, P.; Wang, S.-W.; Kong, W.; Wen, L.-R. J. Org. Chem. 2012, 77, 8956. (i) Yu, F.-C.; Yan, S.-J.; Hu, L.; Wang, Y.-C.; Lin, J. Org. Lett. 2011, 13, 4782. (j) Wen, L.-R.; Liu, C.; Li, M.; Wang, L.-J.J. Org. Chem. 2010, 75,7605 .

(20) (a) Cui, B.-D.; Zuo, J.; Zhao, J.-Q.; Zhou, M.-Q.; Wu, Z.-J.; Zhang, X.-M.; Yuan, W.-C. J. Org. Chem. 2014, 79, 5305. (b) Matiadis, D.; Igglessi-Markopoulou, O.; McKee, V.; Markopoulos, J. Tetrahedron 2014, 70, 2439. (c) Conway, P. A.; Devine, K.; Paradisi, F. Tetrahedron 2009, 65, 2935. (d) Sun, W.; Zhu, G.; Wu, C.; Li, G.; Hong, L.; Wang, R. Angew. Chem. Int. Ed. 2013, 52, 8633. (e) Cui, B.-D.; Zuo, J.; Zhao, J.-Q.; Zhou, M.-Q.; Wu, Z.-J.; Zhang, X.-M.; Yuan, W.-C. J. Org. Chem. 2014, 79, 5305. (f) Melhado, A. D.; Amarante, G. W.; Wang, Z. J.; Luparia, M.; Toste, F. D. J. Am. Chem. Soc. 2011, 133, 3517. (g) Rodríguez-Docampo, Z.; Quigley, C.; Tallon, S.; Connon, S. J. J. Org. Chem. 2012, 77, 2407. (h) Trost, B. M.; Czabaniuk, L. C. J. Am. Chem. Soc. 2012, 134, 5778.

(21) (a) Bernabe, M.; Cuevas, O.; Fernandez-Alvarez, E. Synthesis 1977, 191. (b) Arenal, I.; Bernabe, M.; Cuevas, O.; FernandezAlvarez, E. Tetrahedron 1983, 39, 1387. (c) Bernabe, M.; Cuevas, O.; Fernandez-Alvarez, E. Eur. J. Med. Chem. 1979, 14, 33. (d) Bernabe, M.; Cuevas, O.; Fernandez-Alvarez, E. Tetrahedron Lett. 1977, 18, 895.

(22) Ziyaei Halimehjani, A.; Khoshdoun, M. J. Org. Chem. 2016, 81, 5699.

(23) (a) Ziyaei Halimehjani, A.; Hosseinkhany, S. Synthesis 2015, 47, 3147. (b) Ziyaei Halimehjani, A.; Alaei, M. A.; Soleymani Movahed, F.; Jomeh, N.; Saidi, M. R. J. Sulf. Chem. 2016, 37, 529. (c) Schlüter, T.; Ziyaei Halimehjani, A.; Wachtendorf, D.; Schmidtmann, M.; Martens, J. ACS Comb. Sci. 2016, 18, 456. (d) Ziyaei Halimehjani, A.; Hasani, L.; Alaei, M. A.; Saidi, M. R. Tetrahedron Lett. 2016, 57, 883. (e) Ziyaei Halimehjani, A.; Hajiloo Shayegan, M.; Hashemi, M. M.; Notash, B. Org. Lett. 2012, 14, 3838. (f) Ziyaei Halimehjani, A.; Martens, J.; Schluter, T. Tetrahedron 2016, 72, 3958.

(24) CCDC 1570967 contains the supplementary crystallographic data for this paper. The data can be obtained free of charge from The Cambridge Crystallographic Data Centre via www.ccdc.cam.ac.uk/structures.

(25) Zhao, M. X.; Wang, M. X.; Huang, Z. T. Tetrahedron 2002, 58, 1309. 